\title{
METABOLIC EFFECTS OF UNILATERAL LESION OF THE SUBSTANTIA NIGRA ${ }^{1}$
}

\author{
G. F. WOOTEN ${ }^{2}$ AND R. C. COLLINS
}

Departments of Neurology and Pharmacology, Washington University School of Medicine, St. Louis, Missouri 63110

\begin{abstract}
Regional brain glucose utilization following unilateral lesion of the substantia nigra in rat was studied by $\left[{ }^{14} \mathrm{C}\right]$-2-deoxyglucose autoradiography. Substantia nigra lesions were performed by perinigral injections of 6 hydroxydopamine (6-OHDA) $\cdot \mathrm{HBr}, 6 \mu \mathrm{g}$, in rats pretreated 30 min earlier with desmethylimipramine (DMI), $25 \mathrm{mg} / \mathrm{kg}$, subcutaneously. The lesion produced extensive destruction of the ipsilateral substantia nigra pars compacta and a greater than $99 \%$ reduction in dopamine concentration in the ipsilateral striatum.

Pretreatment with DMI prevented any reduction in the concentration of norepinephrine in ipsilateral forebrain structures. Glucose utilization was increased in the ipsilateral globus pallidus at 11, 21, 53, and 104 days after substantia nigra lesion with the largest increase (about 140\% of control) occurring at 21 days post-lesion. In addition, glucose utilization in ipsilateral lateral habenular nucleus was increased at each of the above time points. No changes in glucose utilization were noted in frontal cortex, striatum, subthalamic nucleus, entopeduncularis, or ventral tier nuclei of the thalamus. These results suggest that lesion of the substantia nigra with depletion of striatal dopamine content results in disinhibition of some striatal, and perhaps olfactory cortical, efferents producing increased metabolism and glucose utilization in terminal fields within the globus pallidus and lateral habenular nucleus.
\end{abstract}

Degeneration of nigrostriatal dopaminergic neurons with depletion of striatal dopamine appears to be the critical pathologic process resulting in the signs and symptoms of Parkinson disease (Hornykiewicz, 1972). This hypothesis is supported by the observation that administration of $\mathrm{L}$-dopa, which is converted to dopamine in the brain, causes a dramatic improvement in patients with idiopathic parkinsonism (Cotzias et al., 1967). How this relatively discrete lesion alters the normal function of the local neuronal circuits of the basal ganglia is not well understood.

It has been possible in recent years to produce highly selective lesions of the substantia nigra in experimental animals by means of intracerebral injections of the catecholamine neurotoxin, 6-hydroxydopamine (6-OHDA) (Ungerstedt, 1971). Further, with the development of the $\left[{ }^{14} \mathrm{C}\right]$-2-deoxyglucose autoradiographic method of Sokoloff et al. (1977), it has become possible to measure simultaneously the rate of glucose utilization or "functional metabolism" in individual structures within the

\footnotetext{
${ }^{1}$ This work was supported by research grants from the American Parkinson Disease Association, the Institute for Medical Education and Research of the City of St. Louis, and National Institutes of HealthNational Institute of Neurological and Communicative Disorders and Stroke Grant 1-P01-14834-1. G. F. W. is a George C. Cotzias Fellow of the American Parkinson Disease Association.

${ }^{2}$ To whom correspondence should be addressed at Department of Neurology, Box 8111, Washington University School of Medicine, 660 South Euclid, St. Louis, MO 63110.
}

brain. We have combined these two methodologies in an effort to understand better the functional effects of unilateral dopaminergic denervation on the highly interrelated circuits within the basal ganglia. We find that unilateral destruction of the substantia nigra in rat results in a large increase in glucose utilization in the ipsilateral globus pallidus and lateral habenular nucleus.

\section{Materials and Methods}

Preparation of animals. Male Sprague-Dawley rats weighing 300 to $350 \mathrm{gm}$ were anesthetized with ketamine, $120 \mathrm{mg} / \mathrm{kg}$, subcutaneously, pretreated with desmethylimipramine (DMI), $25 \mathrm{mg} / \mathrm{kg}$, subcutaneously, $30 \mathrm{~min}$ prior to 6-OHDA injection (Breese and Traylor, 1971), and placed in a Kopf stereotaxic frame. 6-Hydroxydopamine hydrobromide, $3 \mathrm{mg} / \mathrm{ml}$, and ascorbic acid, $1 \mathrm{mg} /$ $\mathrm{ml}$, were dissolved in normal saline, maintained in the dark at $4^{\circ} \mathrm{C}$, and loaded into a 30 gauge stainless steel cannula just prior to intracerebral injection. The cannula tip was lowered through a burr hole in the skull to a site near the substantia nigra with the following coordinates: incisor bar, 0; AP, $3.4 \mathrm{~mm}$; $\mathrm{ML}, 2.0 \mathrm{~mm}$; and $\mathrm{DV}, 2.8 \mathrm{~mm}$ (about $7.5 \mathrm{~mm}$ below the dural touch point). One minute after placement of the cannula, the injection was begun at the rate of $0.5 \mu \mathrm{l} / \mathrm{min}$. The total volume injected was $2.0 \mu \mathrm{l}$ (a total dose of $6 \mu \mathrm{g}$ of 6 -hydroxydopamine as the hydrobromide salt). Five minutes after completion of the injection, the cannula was removed. Ten days after the 6-OHDA injections, rats were placed in individual cages, 
injected with apomorphine $\mathrm{HCl}, 0.5 \mathrm{mg} / \mathrm{kg}$, subcutaneously, and observed for rotational behavior for $20 \mathrm{~min}$ (Ungerstedt, 1971). Only rats that exhibited consistent contralateral turning were used in subsequent experiments.

Assay of brain regions for dopamine and norepinephrine content. Two weeks after injection of 6-OHDA, rats were killed by cervical dislocation and their brains were removed rapidly and placed on a chilled stainless steel surface. The brain was dissected under magnification into the following regions: striatum, olfactory cortex-nucleus accumbens, septum, frontal cortex, and hypothalamus. The tissues were frozen on dry ice, weighed, and homogenized in $\mathrm{HClO}_{4}, 0.2 \mathrm{~N}$, containing reduced glutathione, $5 \mathrm{~mm}$, to make a $1 \%$ homogenate. Aliquots of the homogenates then were assayed for dopamine and norepinephrine content by the method of Cheng and Wooten (1980).

$\left[{ }^{14} \mathrm{C}\right]$-2-Deoxyglucose autoradiography. $\left[{ }^{14} \mathrm{C}\right]-2$-Deoxyglucose autoradiography was performed according to the method of Sokoloff et al. (1977). At various times (from 11 to 104 days) after the 6-OHDA lesion of the substantia nigra, $\left[{ }^{14} \mathrm{C}\right]$-2-deoxyglucose, 50 to $60 \mu \mathrm{Ci} / \mathrm{kg}$ of body weight, was injected into awake freely moving rats via a venous cannula placed 3 to $4 \mathrm{hr}$ earlier under halothane anesthesia. Forty-five minutes later, the animals were sacrificed with intravenous sodium pentobarbital, perfused with sodium cacodylate, $0.2 \mathrm{~m}, \mathrm{pH} 7.3$, followed by paraformaldehyde, $3.3 \%$, in the same buffer. The brain was removed, rapidly frozen in liquid Freon, and mounted on brass chucks. Duplicate $20-\mu \mathrm{m}$ sections were taken every $200 \mu \mathrm{m}$, mounted on cover glasses, and exposed to Kodak SB-5 x-ray film for 5 days. Selected sections were then taken for thionine stains and densitometry was performed on the autoradiographs. All optical density (OD) measurements except for those in the quantitative study consist of a ratio of $\mathrm{OD}$ in region divided by OD of corpus callosum. Quantitative $\left[{ }^{14} \mathrm{C}\right]-2$ - deoxyglucose autoradiography was also performed according to the method of Sokoloff et al. (1977).

\section{Results}

Effects of substantia nigra lesion with DMI pretreatment on regional brain norepinephrine and dopamine content. In a series of preliminary experiments, intracerebral injections of 6-OHDA into the region of substantia nigra were made with doses varying from 2 to $12 \mu \mathrm{g}$ as hydrobromide salt. Using histological criteria, it was determined that a dose of $6 \mu \mathrm{g}$ produced the most extensive lesion of the substantia nigra pars compacta with the least amount of nonspecific tissue damage as evidenced by a glial reaction in the injection site just dorsal to the pars compacta. Figure 1 illustrates the degree of neuronal loss in substantia nigra 4 weeks after 6-OHDA injection of $6 \mu \mathrm{g}$.

Two groups of four rats each were injected intracerebrally with $6-\mathrm{OHDA}, 6 \mu \mathrm{g}$. One group received desmethylimipramine $\mathrm{HCl}$ (DMI), $25 \mathrm{mg} / \mathrm{kg}$, subcutaneously 30 min before the 6-OHDA lesion while the control group received a subcutaneous injection of saline. Two weeks later, the animals were killed and regional brain dopamine and norepinephrine content was determined. The results are shown in Table I. 6-OHDA treatment resulted in a large reduction in dopamine content of each ipsilateral structure independent of DMI pretreatment. However, DMI pretreatment completely blocked the reduction in norepinephrine content of ipsilateral structures seen in the rats not pretreated with DMI.

Thus, the injection of 6-OHDA resulted in extensive neuronal destruction of substantia pars compacta neurons and a large $(>99 \%)$ reduction in ipsilateral striatal dopamine content. In addition, pretreatment with DMI prior to the 6-OHDA injection appeared to protect the ascending noradrenergic axons in the adjacent median forebrain bundle from the toxic effects of 6-OHDA.

Effects of substantia nigra lesion on regional brain

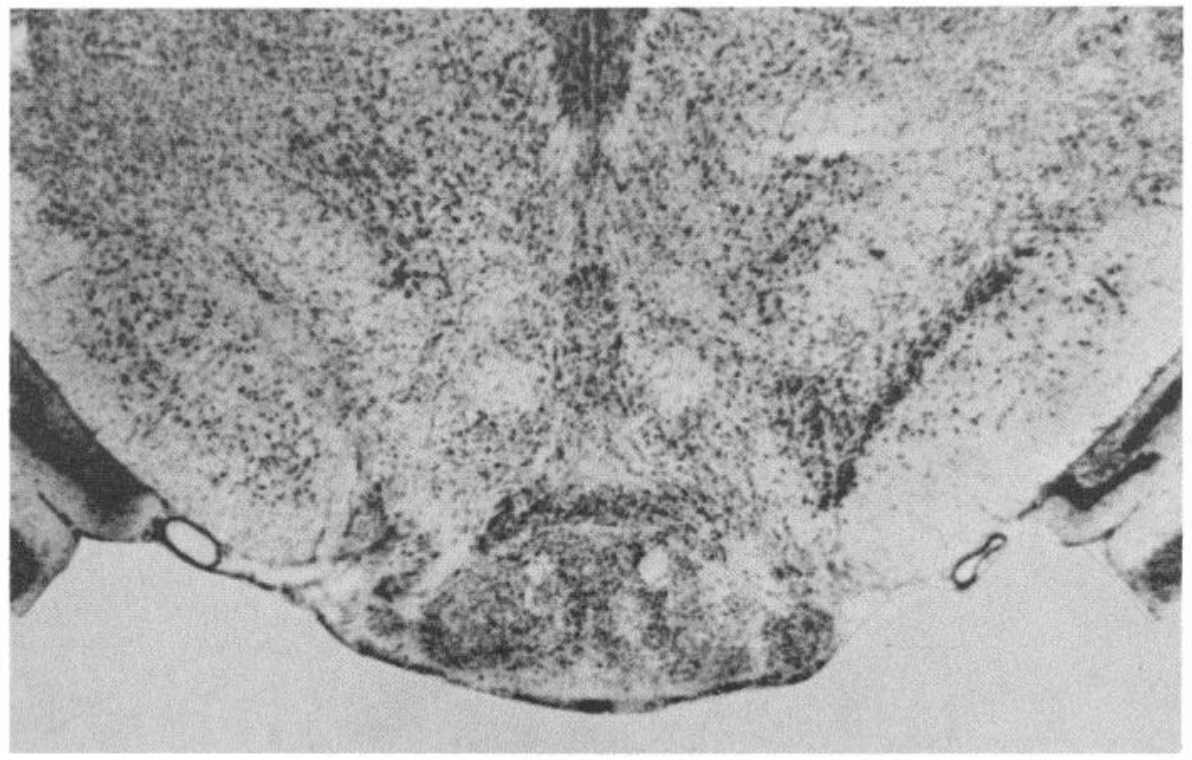

Figure 1. Thionine-stained section of the ventral portion of the mesencephalon 4 weeks after unilateral injection of 6-OHDA on the left side. Note the marked cell loss in the substantia nigra pars compacta. 
TABLE I

Substantia nigra lesion with 6-OHDA

Each number represents the mean \pm SEM.

\begin{tabular}{|c|c|c|c|c|c|c|}
\hline \multirow{2}{*}{ Brain Region } & \multicolumn{3}{|c|}{ Dopamine } & \multicolumn{3}{|c|}{ Norepinephrine } \\
\hline & Lesion & Control & Ratio & Lesion & Control & Ratio \\
\hline & \multicolumn{6}{|c|}{ No DMI Pretreatment $(n=4)$} \\
\hline & \multicolumn{2}{|c|}{$\mu g / g m$} & \multicolumn{4}{|c|}{$\mu g / g m$} \\
\hline Striatum & $0.09 \pm 0.06$ & $8.16 \pm 0.71$ & $0.01^{a}$ & $0.02 \pm 0.001$ & $0.12 \pm 0.01$ & $0.17^{a}$ \\
\hline Olfactory-accumbens & $0.36 \pm 0.08$ & $4.66 \pm 0.46$ & $0.08^{a}$ & $0.10 \pm 0.03$ & $0.33 \pm 0.09$ & $0.30^{a}$ \\
\hline Septum & $0.26 \pm 0.06$ & $1.18 \pm 0.22$ & $0.22^{a}$ & $0.16 \pm 0.03$ & $0.46 \pm 0.03$ & $0.35^{a}$ \\
\hline Frontal cortex & $0.03 \pm 0.03$ & $0.19 \pm 0.08$ & $0.16^{a}$ & 0 & $0.20 \pm 0.02$ & \\
\hline \multirow[t]{3}{*}{ Hypothalamus } & $0.34 \pm 0.03$ & $0.50 \pm 0.08$ & $0.68^{a}$ & $0.59 \pm 0.10$ & $1.32 \pm 0.27$ & $0.45^{a}$ \\
\hline & \multicolumn{6}{|c|}{ With DMI Pretreatment $(n=4)$} \\
\hline & \multicolumn{2}{|c|}{$\mu g / g m$} & \multicolumn{4}{|c|}{$\mu g / g m$} \\
\hline Striatum & $0.03 \pm 0.001$ & $9.50 \pm 0.65$ & $0.003^{a}$ & $0.13 \pm 0.02$ & $0.11 \pm 0.02$ & $1.18^{b}$ \\
\hline Olfactory-accumbens & $0.82 \pm 0.20$ & $4.60 \pm 0.32$ & $0.17^{a}$ & $0.28 \pm 0.03$ & $0.25 \pm 0.04$ & $1.12^{b}$ \\
\hline Septum & $0.46 \pm 0.06$ & $1.00 \pm 0.46$ & $0.46^{a}$ & $0.38 \pm 0.03$ & $0.43 \pm 0.03$ & $0.88^{b}$ \\
\hline Frontal cortex & 0 & $0.15 \pm 0.02$ & & $0.17 \pm 0.03$ & $0.18 \pm 0.02$ & $0.94^{b}$ \\
\hline Hypothalamus & $0.38 \pm 0.10$ & $0.50 \pm 0.06$ & $0.76^{b}$ & $1.32 \pm 0.10$ & $1.20 \pm 0.12$ & $1.10^{b}$ \\
\hline
\end{tabular}

${ }^{a}$ Lesion side differs from control $(p<0.01)$.

${ }^{b}$ No difference between lesion and control.

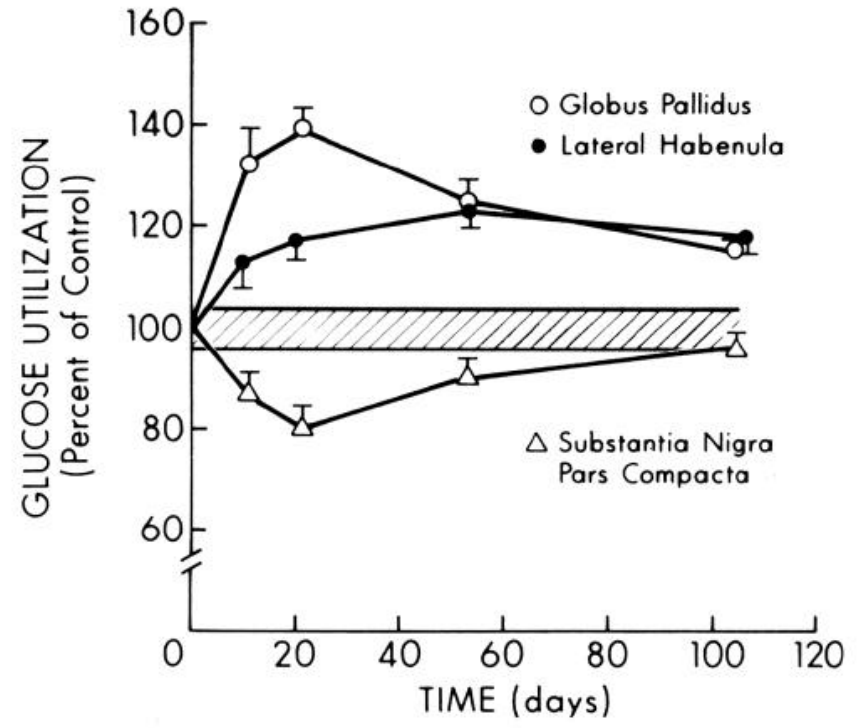

Figure 2. Regional brain glucose utilization in globus pallidus, lateral habenular nucleus, and substantia nigra pars compacta as a function of time after 6-OHDA lesion of the substantia nigra. The points at 11 and 21 days represent the mean \pm SD of four animals while the points at 53 and 104 days are the mean $\pm \mathrm{SD}$ of three animals. The horizontal striped bar represents the contralateral control mean $\pm 2 \mathrm{SD}$. Glucose utilization as a percentage of control represents the ratio of optical density in each region divided by the mean optical density of several white matter areas in turn divided by the same ratio in the contralateral, unlesioned hemisphere. All points except for the substantia nigra pars compacta at 104 days differ from control by at least the $p<0.05$ confidence limit.

glucose utilization. Regional brain glucose utilization was studied 11,21, 53, and 104 days after substantia nigra lesion. The regions showing changes compared to the contralateral control side are shown in Figure 2. There was a large increase in glucose utilization in ipsilateral globus pallidus at 11 and 21 days after lesion which was

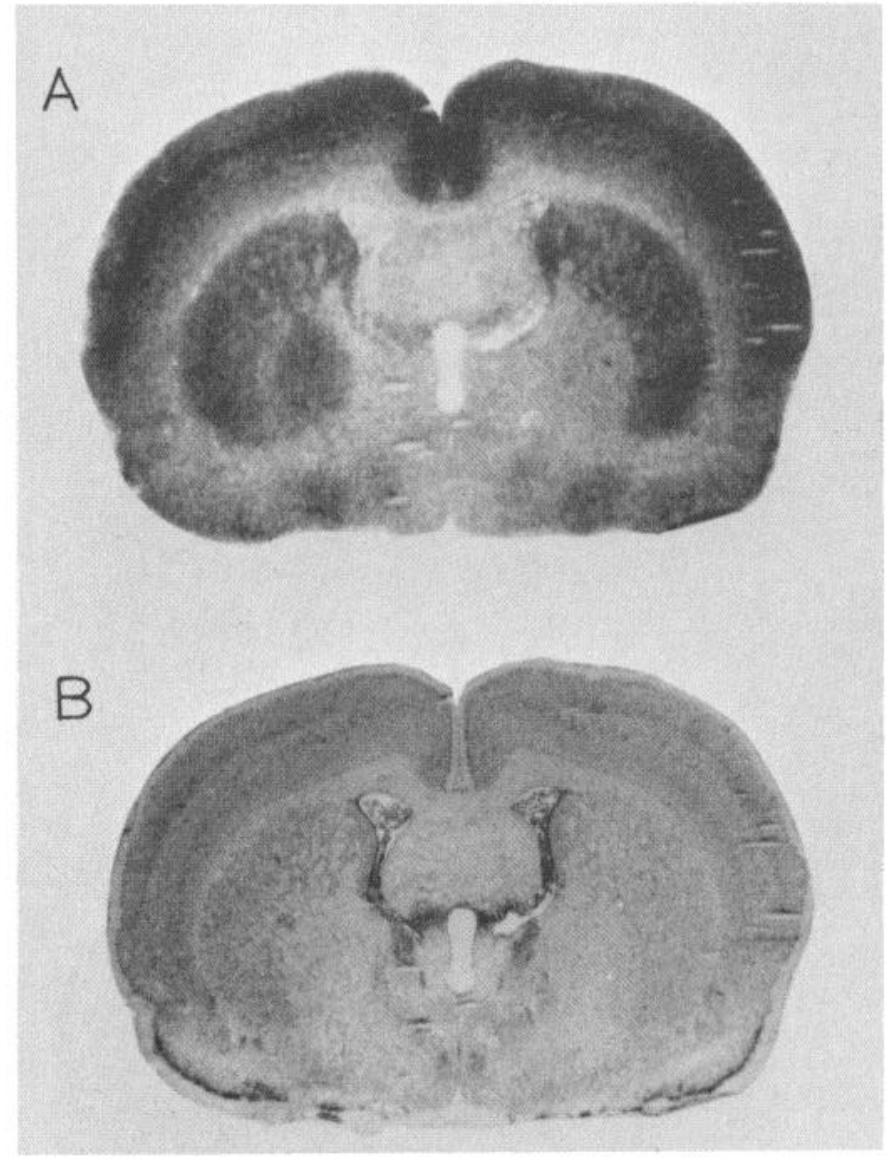

Figure 3. $A,\left[{ }^{14} \mathrm{C}\right]-2$-Deoxyglucose autoradiograph at the frontal plane of the globus pallidus 3 weeks after 6-OHDA lesion of the left substantia nigra. $B$, Thionine-stained section which produced the autoradiographic image in $A$.

less apparent at 53 and 104 days. Also, glucose utilization was increased in the lateral portion of the ipsilateral lateral habenular nucleus at each time point studied. Finally, glucose utilization was transiently reduced in the 
ipsilateral substantia nigra pars compacta. Autoradiographs of each region 3 weeks after 6-OHDA lesion are depicted in Figure 3 (globus pallidus), Figure 4 (lateral habenular nucleus), and Figure 5 (substantia nigra pars compacta). No asymmetry was noted in neocortex, striatum, subthalamic nucleus, entopeduncularis, substantia nigra pars reticulata, or ventral tier nuclei of thalamus. These asymmetric changes appeared to be specific consequences of 6-OHDA injection and not due to nonspecific effects of tissue damage from the injection because rats receiving intracerebral injections of similar volumes of normal saline and ascorbic acid $(2 \mu \mathrm{g})$ showed no histological damage of ipsilateral substantia nigra and absolutely no changes (or asymmetries) in regional brain glucose utilization.

Table II summarizes the results of three rats studied by quantitative $\left[{ }^{14} \mathrm{C}\right]$-2-deoxyglucose autoradiography 2 weeks after 6-OHDA injections. These data confirm the selective increase in glucose utilization in ipsilateral globus pallidus and lateral habenular nucleus and decrease in substantia nigra pars compacta. In addition, these data suggest that no change in glucose utilization occurred in other ipsilateral or any contralateral structures compared to naive, unlesioned control rats.

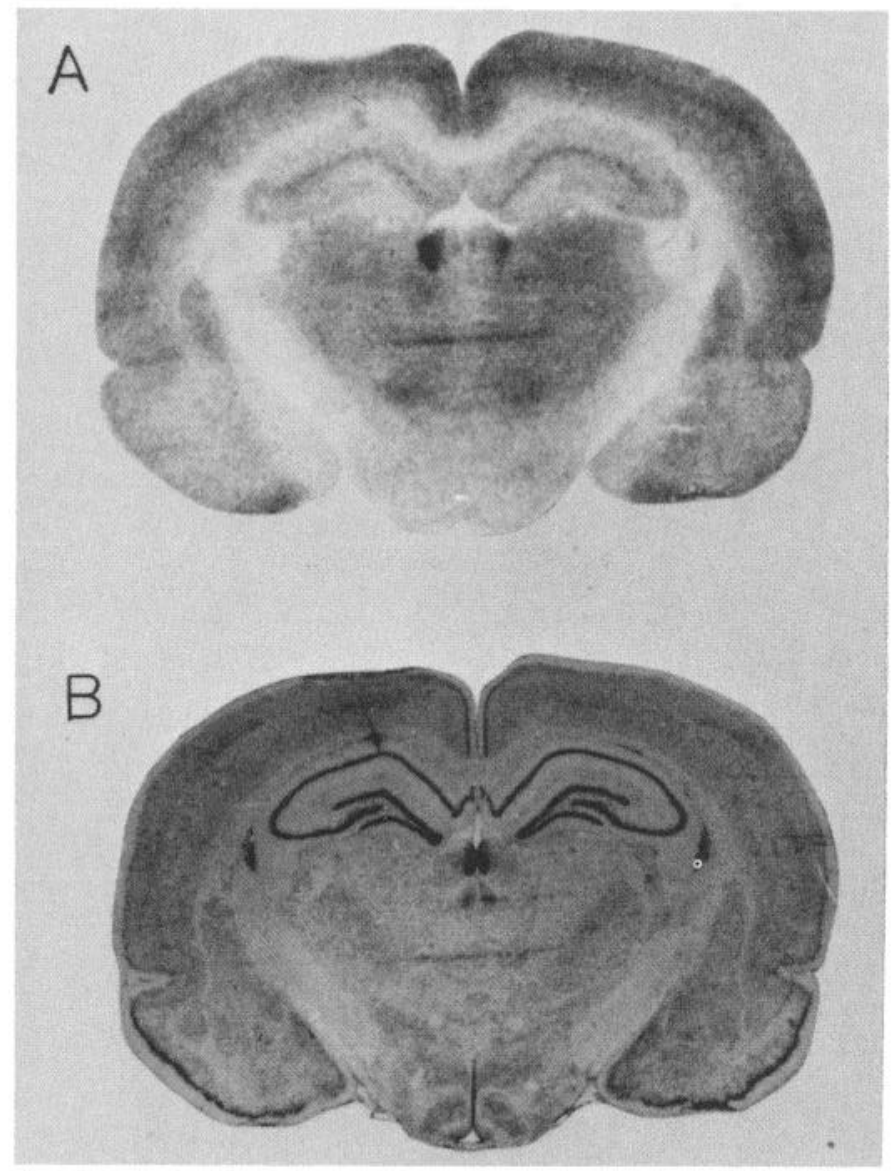

Figure 4. $A,\left[{ }^{14} \mathrm{C}\right]-2$-Deoxyglucose autoradiograph at the frontal plane of the habenula 3 weeks after 6-OHDA lesion of the left substantia nigra. $B$, Thionine-stained section which produced the autoradiographic image in $A$.

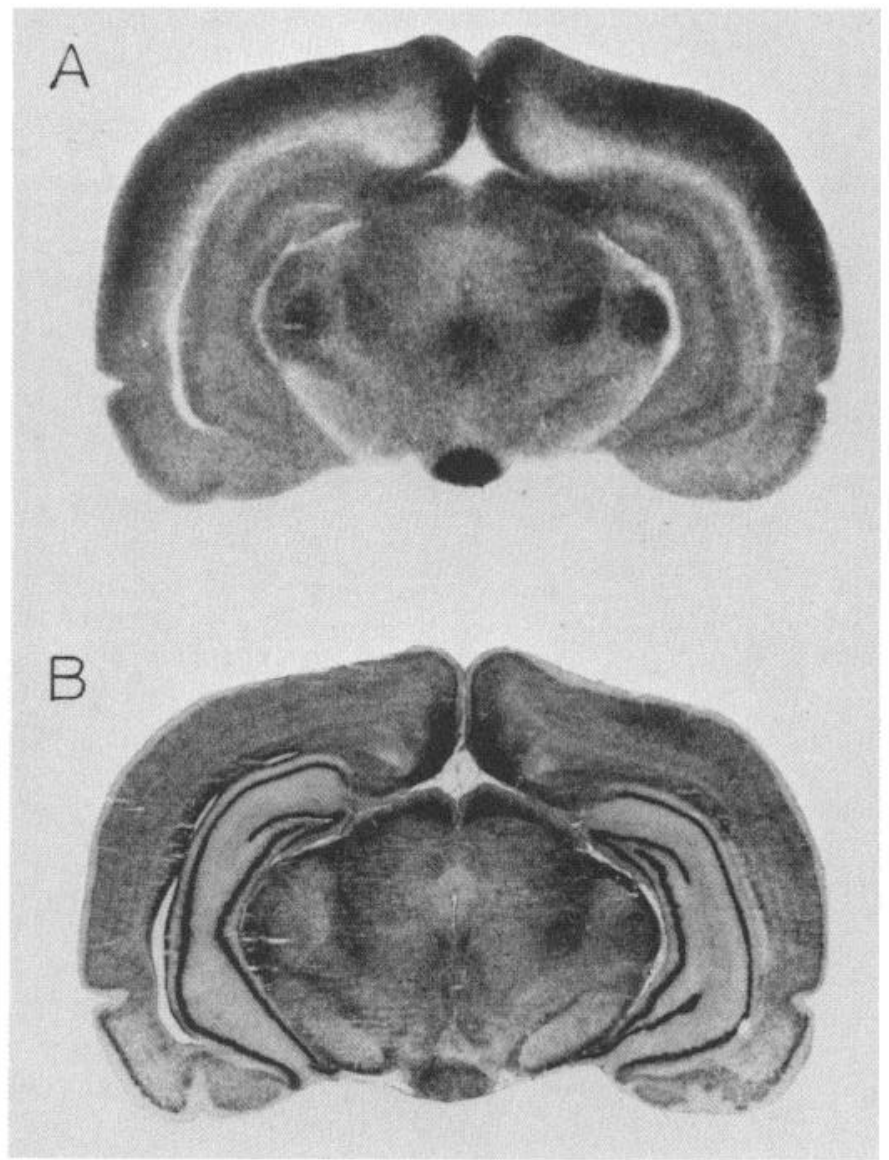

Figure 5. $A,\left[{ }^{14} \mathrm{C}\right]$-2-Deoxyglucose autoradiograph at the frontal plane of the mesencephalon 3 weeks after 6-OHDA lesion of the left substantia nigra. There is a reduction in glucose utilization in the region of the left substantia nigra pars compacta. $B$, Thionine-stained section which produced the autoradiographic image in $A$.

TABLE II

Quantitative $\left[{ }^{14} \mathrm{C}\right]$ deoxyglucose autoradiography in rats 2 weeks after unilateral lesion of substantia nigra

Each number represents the mean \pm 1 SD.

\begin{tabular}{|c|c|c|c|}
\hline \multirow{3}{*}{ Brain Region } & \multicolumn{3}{|c|}{ Glucose Utilization } \\
\hline & \multirow{2}{*}{$\begin{array}{l}\text { Control } \\
(n=5)\end{array}$} & \multicolumn{2}{|c|}{ Lesioned Animals $(n=3)$} \\
\hline & & $\begin{array}{l}\text { Ipsilateral } \\
\text { to Lesion }\end{array}$ & $\begin{array}{l}\text { Contralateral } \\
\text { to Lesion }\end{array}$ \\
\hline & \multicolumn{3}{|c|}{$\mu \mathrm{mol} / \mathrm{gm} / \mathrm{min}$} \\
\hline Frontal cortex & $1.03 \pm 0.21$ & $1.00 \pm 0.13$ & $1.12 \pm 0.11$ \\
\hline Striatum & $1.08 \pm 0.12$ & $1.08 \pm 0.06$ & $1.15 \pm 0.05$ \\
\hline Globus pallidus & $0.58 \pm 0.15$ & $0.89 \pm 0.04^{a}$ & $0.60 \pm 0.07$ \\
\hline Subthalamic nucleus & $1.06 \pm 0.15$ & $0.91 \pm 0.07$ & $0.89 \pm 0.15$ \\
\hline $\begin{array}{l}\text { Lateral habenular } \\
\text { nucleus }\end{array}$ & $1.33 \pm 0.22$ & $2.01 \pm 0.32^{a}$ & $1.37 \pm 0.13$ \\
\hline $\begin{array}{l}\text { Substantia nigra } \\
\text { pars compacta }\end{array}$ & $0.82 \pm 0.11$ & $0.69 \pm 0.04^{b}$ & $0.81 \pm 0.05$ \\
\hline $\begin{array}{r}\text { Substantia nigra } \\
\text { pars reticulata }\end{array}$ & $0.54 \pm 0.11$ & $0.49 \pm 0.04$ & $0.51 \pm 0.15$ \\
\hline
\end{tabular}

${ }^{a}$ Differs from control $p<0.01$.

${ }^{b}$ Differs from control $p<0.05$. 


\section{Discussion}

To conclude from these studies that destruction of the ascending nigrostriatal dopaminergic pathway results in an increase in glucose utilization in ipsilateral globus pallidus and lateral habenular nucleus requires a high degree of confidence in the completeness and specificity of the lesion. That the lesion was complete was evidenced by the histological picture of extensive neuronal loss in ipsilateral substantia nigra pars compacta and a greater than $99 \%$ reduction in dopamine content of the ipsilateral striatum. Histological evidence for specificity rested on the apparent lack of damage to any adjacent nuclear groups including red nucleus and subthalamic nucleus. However, some damage to axons in passage cannot be unequivocally excluded. Biochemical specificity was conferred to a great extent by 6 -hydroxydopamine which is concentrated in and selectively destroys catecholamine neurons (Kostrzewa and Jacobowitz, 1974). Pretreatment of rats with DMI prior to 6-OHDA injections protected noradrenergic axons in the nearby median forebrain bundle presumably by blocking the uptake of 6-OHDA into these neurons (Breese and Traylor, 1971). That the changes in glucose utilization were a consequence of nonspecific damage by the injections, or perhaps the ascorbic acid, was excluded by observations in control rats injected with the same volume of saline containing the same concentration of ascorbate as the experimental animals. Based on the above considerations, we can conclude with a high degree of confidence that increased glucose utilization in globus pallidus and lateral habenular nucleus resulted from extensive and specific destruction of the nigrostriatal dopamine pathway.

The predominant projection site of dopaminergic neurons from the substantia nigra pars compacta is the striatum (Fuxe, 1965). Further, there is evidence suggesting that dopamine is an inhibitory neurotransmitter (Siggins et al., 1976). It would be reasonable then to predict that a physiologic consequence of removal of the dopaminergic innervation of the striatum would be net disinhibition of denervated neuronal elements. Why, then, was glucose utilization increased in globus pallidus but not striatum? We would suggest that the explanation derives from observations indicating that the brain structures showing the greatest increase in glucose utilization following stimulation are nerve endings and dendrites where surface-to-volume ratios are very high and the energy utilized to pump ions is expended at the highest rate per unit volume of tissue (Mata et al., 1980). The major projection of striatum is to globus pallidus (Cowan and Powell, 1966; Nauta and Mehler, 1966). If striatal neurons projecting to globus pallidus are disinhibited, then one would expect to see increased firing and increased glucose utilization primarily in the terminal fields within globus pallidus. Alternatively, it is theoretically possible that increased inhibition with hyperpolarization could lead to an increase in the rate of local glucose utilization over base line rates. Direct evidence to support these speculations cannot be obtained by $\left[{ }^{14} \mathrm{C}\right]-2$-deoxyglucose autoradiography, however, because the precise structures (i.e., cell bodies, dendrites, nerve endings) cannot be resolved due to the relatively high energies of the ${ }^{14} \mathrm{C} \beta$ emissions. Another contributing factor may be the removal of direct dopamine projections to pallidal neurons, but the existence of this projection is somewhat controversial (Mettler, 1970; Carpenter and Peter, 1972).

Recent physiological observations by Filion (1979) seem consistent with our observations of increased glucose utilization in globus pallidus following nigrostriatal dopamine lesions. Filion recorded spontaneous activity of pallidal neurons in monkeys following both lesions of the nigrostriatal pathway and chronic administration of dopamine antagonists. In each paradigm, pallidal neurons fired in bursts continuously during sleep, movement, or quiet wakefulness, whereas pallidal recordings from unlesioned animals revealed discharges interrupted by long periods of silence.

It is of interest that we found no changes in glucose utilization in several other structures within the local circuits of the basal ganglia that are linked in series with the striatum and globus pallidus. These structures include subthalamic nucleus, entopeduncularis, and ventral tier nuclei of the thalamus (Nauta, 1979). It is possible that physiologic changes did occur in these structures but were below the limits of resolution of our method.

Speculation regarding the mechanism of the increase in glucose utilization in the lateral habenular nucleus (LHN) following substantia nigra lesion also derives directly from anatomical considerations. Within the limits of resolution, it appeared that the lateral portion of the LHN was the area where glucose utilization was highest. Herkenham and Nauta (1977) demonstrated that this region of the LHN receives afferents primarily from the entopeduncularis. The entopeduncularis, in turn, receives direct projections from striatum and from the subthalamic nucleus. Further, the medial portion of the LHN receives its major afferent input from olfactory cortex (Herkenham and Nauta, 1977). It is perhaps of significance that our lesion of substantia nigra resulted in a large reduction in dopamine concentration in olfactory corlex (see Table I), indicating that, in addition to nigral neurons, more medially located A-10 dopaminergic neurons were lesioned by the 6-OHDA injections as well (Ungerstedt, 1971). Thus, the lateral habenular nucleus receives both mono- (from olfactory cortex) and disynaptic (from striatum via entopeduncularis) projections from structures deprived of their rather dense dopamine innervation as a consequence of the nigral lesion. Also, more recent evidence indicates the existence of a direct projection from the ventral tegmental area to the lateral habenular nucleus (Beckstead et al., 1979). This projection could be dopaminergic and hence could possibly be lesioned by our 6 -hydroxydopamine injection. We speculate that, like the changes in globus pallidus, the increased glucose utilization in LHN following nigral lesion resulted from increased activity in disinhibited afferents arising in entopeduncularis and perhaps olfactory cortex. Further, there may be loss of direct inhibition via lesion of dopaminergic projections from the ventral tegmental area to LHN. Other evidence of a functional role for dopamine in controlling the metabolic rate in the LHN has been presented by McCulloch et al. (1980). Using 
$\left[{ }^{14} \mathrm{C}\right]$-2-deoxyglucose autoradiography, they found that apomorphine decreased and haloperidol increased glucose utilization in LHN.

In earlier studies, cerebral glucose utilization in rats following electrolytic lesions of lateral hypothalamus and 6-OHDA lesions of substantia nigra was described (Schwartz et al., 1976; Schwartz, 1978). These authors studied animals 3 days after lesioning and found that glucose utilization was reduced in a variety of ipsilateral forebrain structures including striatum. No change was noted in globus pallidus but glucose utilization was increased in the habenula by $8 \%$ above control rates. Studying these animals at 3 days may have been too early, as Ungerstedt has reported that several days are required for full depletion of dopamine from striatum after lesion of the nigrostriatal pathway (Ungerstedt, 1971). In addition, the electrolytic lesions destroyed the entire median forebrain bundle, DMI pretreatment was not used in the 6-OHDA-lesioned animals to protect noradrenergic pathways, and no measurement of striatal dopaminergic markers or histological evidence of completeness of the 6-OHDA lesion was reported. Thus, we find it difficult to compare meaningfully our results to those of Schwartz (1978).

In a more recent study, Sagar and Snodgrass (1980) studied forebrain 2-deoxyglucose retention following 6OHDA lesion. Their rats were not pretreated with DMI to protect noradrenergic fibers but all other aspects of their study are comparable with our own; these aspects include careful histological characterization of the lesion site, measurement of tyrosine hydroxylase activity and dopamine concentration in striatum after lesion, and carefully studied control animals injected with vehicle. Insofar as their results were reported, there were no discrepancies with our own. They found no changes as a result of substantia nigra lesion in glucose utilization in striatum, parietal cortex, or frontal cortex ipsilateral to the lesion. Unaccountably, they did not comment on the globus pallidus or lateral habenular nucleus. Thus, we cannot agree with their conclusion that lesions of the nigrostriatal dopaminergic pathway have only modest effects on 2-deoxyglucose retention by forebrain structures. Also, we found no changes in anatomy, behavior, glucose metabolism, or dopamine concentration as a result of vehicle injections into brain.

Our observation that the increased glucose utilization in the globus pallidus seen at 11 and 21 days after lesion appears to be attenuated at 53 and 104 days suggests that some adaptive change occurred over a period of several months. This return toward control rates of glucose utilization is not accompanied by dopaminergic re-innervation, nor by attenuation of asymmetric motor responses to treatment with apomorphine or amphetamine. The mechanism of this time-dependent change remains to be elucidated.

Selective lesion of the nigrostriatal dopamine pathway resulted in a large increase in glucose utilization in ipsilateral globus pallidus. Since this lesion reproduces the major biochemical and pathological changes found in the brains of patients with Parkinson disease (Hornykiewicz, 1972), one might reasonably speculate that striopallidal neurons are chronically disinhibited, producing an excess of their neurotransmitter in the globus pallidus. There is evidence that a prominent striopallidal projection is enkephalinergic (Elde et al., 1976). It is thus of much interest that Vardi et al. (1979) have recently reported that administration of the opiate antagonist naloxone to patients with Parkinson disease resulted in a significant clinical improvement.

\section{References}

Beckstead, R. M., V. B. Domesick, and W. J. H. Nauta (1979) Efferent connections of the substantia nigra and ventral tegmental area in the rat. Brain Res. 175: 191-217.

Breese, G. R., and T. D. Traylor (1971) Depletion of brain noradrenalin and dopamine by 6 -hydroxydopamine. $\mathrm{Br}$. J. Pharmacol. 42: 88-99.

Carpenter, M. B., and P. Peter (1972) Nigrostriatal and nigrothalamic fibers in the rhesus monkey. J. Comp. Neurol. 144: 93-116.

Cheng, C. H., and G. F. Wooten (1980) A highly sensitive radioenzymatic assay for simultaneous estimation of norepinephrine, dopamine, and epinephrine. J. Pharmacol. Methods 3: $333-344$.

Cotzias, G. C., M. H. Van Woert, and L. M. Schiffer (1967) Aromatic amino acids and modification of parkinsonism. $\mathrm{N}$. Engl. J. Med. 276: 374-379.

Cowan, W. M., and T. P. S. Powell (1966) Striopallidal projection in the monkey. J. Neurol. Neurosurg. Psychiatry 29: $426-439$.

Elde, R., T. Hokfelt, O. Johansson, and L. Terenius (1976) Immunohistochemical studies using antibodies to leucineenkephalin: Initial observations on the nervous system of the rat. Neuroscience 1: 349-351.

Filion, M. (1979) Effects of interruption of the nigrostriatal pathway and of dopaminergic agents on the spontaneous activity of globus pallidus neurons in the awake monkey. Brain Res. 178: 425-441.

Fuxe, K. (1965) Evidence for the existence of monoamine neurons in the central nervous system-IV. Distribution of monoamine terminals in the central nervous system. Acta. Physiol. Scand. (Suppl. 247) 64: 37-85.

Herkenham, M., and W. J. H. Nauta (1977) Afferent connections of the habenular nuclei in the rat. A horseradish peroxidase study, with a note on the fiber of passage problem. J. Comp. Neurol. 173: 123-146.

Hornykiewicz, O. (1972) Neurochemistry of parkinsonism. In Handbook of Neurochemistry 7, A. Lajtha, ed., pp. 465-501, Plenum Press, New York.

Kostrzewa, R. M., and D. M. Jacobowitz (1974) Pharmacological actions of 6-hydroxydopamine. Pharmacol. Rev. 26: 199288.

Mata, M., D. J. Fink, H. Gainer, C. B. Smith, L. Davidsen, H. Savaki, W. J. Schwartz, and L. Sokoloff (1980) Activitydependent energy metabolism in rat posterior pituitary primarily reflects sodium pump activity. J. Neurochem. 34: 213215.

McCulloch, J., H. E. Savaki, and L. Sokoloff (1980) Influence of dopaminergic systems on the habenular nucleus. Brain Res. 194: 117-124.

Mettler, F. A. (1970) Nigrofugal connections in the primate brain. J. Comp. Neurol. 138: 291-320.

Nauta, H. J. W. (1979) A proposed conceptual reorganization of the basal ganglia and telencephalon. Neuroscience 4: 18751881.

Nauta, W. J. H., and W. R. Mehler (1966) Projections of the lentiform nucleus in the monkey. Brain Res. 1: 3-42.

Sagar, S. M., and S. R. Snodgrass (1980) Effects of substantia nigra lesions on forebrain 2-deoxyglucose retention in the rat. Brain Res. 185: 335-348.

Schwartz, W. J. (1978) A role for the dopaminergic nigrostriatal 
bundle in the pathogenesis of altered brain glucose consumption after lateral hypothalamic lesions. Evidence using the ${ }^{14}$ C-labelled deoxyglucose technique. Brain Res. 158: 129-147.

Schwartz, W. J., F. R. Sharp, R. H. Gunn, and E. V. Evarts (1976) Lesions of ascending dopaminergic pathways decrease forebrain glucose utilization. Nature 261: 155-157.

Siggins, G. R., B. J. Hoffer, F. E. Bloom, and U. Ungerstedt (1976) Cytochemical and electrophysiological studies of dopamine in the caudate nucleus. In The Basal Ganglia. Research Publications: Association for Research in Nervous and Mental Disease, M. D. Yahr, ed., Vol. 55, pp. 227-248, Raven Press, New York.
Sokoloff, L., M. Reivich, C. Kennedy, M. H. Des Rosiers, C. S. Patlak, K. D. Pettigrew, O. Sakurada, and M. Shenohara (1977) The ${ }^{14} \mathrm{C}$-deoxyglucose method for the measurement of local cerebral glucose utilization: Theory, procedure, and normal values in the conscious and anesthetized albino rat. J. Neurochem. 28: 897-916.

Ungerstedt, U. (1971) Stereotaxic mapping of monoamine pathways in the rat brain. Acta. Physiol. Scand. (Suppl.) 367: 148.

Vardi, J., S. Fletcher, I. Regev, M. Borenstein, and J. Shapira (1979) The modulatory effect of opiate receptor inhibitor in parkinsonism. Curr. Ther. Res. Clin. Exp. 26: 1015-1018. 